Travaux cités

[1] T. Leżański, Sur les équations du type: $\Psi(x, h)=0(I)$ Studia Math. 59 (1976), $155-175$.

[2] F. Riesz et B. Sz. Nagy Lecons d'analyse fonctionnelle, Budapest 1952.

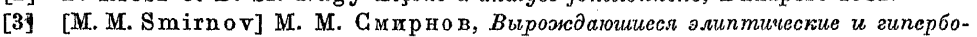
лические уравненил, Москва 1966.

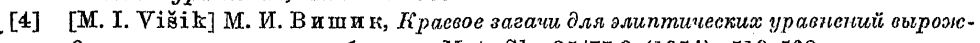
далочихсл на ератище области, Mat. Sb. 35/77.3 (1954), 513-568.

\section{A generalization of the Shinoogaki theorem}

by

\author{
LECH MALIGRANDA (Pozná́)
}

Alustract. There are givon a necessary condition and a sufficient condition for a rearrangement invarianti apace on $(0, l), 0<l \leqslant \infty$ to have an interpolation property for the class of Lipschitiz operators which are of woak typo $(X, X)$ and typo $\left(L^{\infty}, L^{\infty}\right)$ where $X$ is any fixed rearrangement invariant space on $(0, l)$ from the class $\mathscr{L}$.

\$1. Introduction. An operator $T$ that maps a quasi-Banach space $X$ to a quasi-Banach space $Y$ is called a Lipschitz operator if $T 0=0$ and if $\|T f-T g\|_{Y} \leqslant K\|f-g\|_{X}, f, g \in X$ for some $K>0$. The smallest $K$ in this inequality is called the bound of $T$. By $\operatorname{Lip}(X, Y ; K)$ we denote the class of all Lipschitiz operators $T$ from $X$ to $X$ with the bound not exceeding $K$. If $X=Y$, we write briofly $\operatorname{Iip}(X ; K)$ :

Now, let $X$ be a rearrangement invariant space, $\Lambda(X)$ and $M^{*}(X)$ rearrangement invariant Lorentz spaces (see $\S 2)$, and let $\omega$ - $\operatorname{Lip}\left(X ; K_{X}\right)$ $=\operatorname{Lip}\left(\Lambda(X), M^{*}(X) ; K_{X}\right)$ be a class of Lipschitz operators of weal type $(X, X)$ with bound not exceeding $K_{X}$. We say that a Banach space $Y$ such that $Y \subset A(X)+L^{\infty}$ has the interpolation property for the class $\omega-\operatorname{Lip}\left(X ; K_{X}\right) \cap \operatorname{Lip}\left(L^{\infty} ; K_{\infty}\right)$ if, for each $T \in \omega-\operatorname{Lip}\left(X ; K_{X}\right) \cap \operatorname{Lip}\left(L^{\infty} ; K_{\infty}\right)$, $T$ can be considered as a Lipschitz operator from $Y$ into itself.

W. Orlicz [13] has proved that any Orlicz space $L^{\Phi}$ on $(0, l)$ has the interpolation property for the class $\operatorname{Lip}\left(L^{1} ; \boldsymbol{K}_{1}\right) \cap \operatorname{Lip}\left(L^{\infty} ; \boldsymbol{K}_{\infty}\right)$ in the case when $l<\infty$.

G. G. Lorentz and T. Shimogaki [8] have generalized this theorem to the case of any rearrangement invariant space on $(0, l)$. They have also obtained this theorem in the case when $l=\infty$, under an additional assumption of continuity of the norm. In [10] an analogons theorem was obtained for the class $\operatorname{Lip}\left(l^{x} ; K_{1}\right) \cap \operatorname{Lip}\left(e_{0} ; K_{0}\right)$ and for rearrangement invariant sequence spaces. Next, a sufficient condition was given in [11] for a Banach function space $Y$ on $(0, l)$ to have the interpolation property for the class $\operatorname{lip}\left(L^{p} ; K_{p}\right) \cap \operatorname{Lip}\left(L^{\infty} ; K_{\infty}\right)$ and similarly for a Banach sequence space and for the class $\operatorname{Lip}\left(l^{p} ; K_{p}\right) \cap \operatorname{Lip}\left(c_{0} ; K_{0}\right)$. All theorems mentioned above deal with Lipschitz operators of strong type. Interpolation of Lipschitz operators of weak type has been considered by T. Shimogaki [15] who has given the following 
THEoREM A (Shimogalzi). A rearrangement invariant space $Y$ on $(0, l)$ has the interpolation property for the class $\omega$ - $\operatorname{Lip}\left(L^{1} ; K_{1}\right) \cap \operatorname{Lip}\left(L^{\infty} ; K_{\infty}\right)$ if and only if $\alpha(Y)<1$, where $\alpha(Y)$ denotes the upper Boyd of the space $\bar{Y}$. If $l=\infty$, one additionally assumes in the sufficient condition that the norm on $Y$ is continuous.

In this paper, in Section 4, Theorem $\mathrm{A}$ is generalized by giving a nocessary condition and a sufficient condition for a rearrangement invariant space $\boldsymbol{Y}$ to have the interpolation property for the class $\omega-\operatorname{Lip}\left(X ; K_{X}\right) \cap$ $\cap \operatorname{lip}\left(L^{\infty} ; K_{\infty}\right)$, where $X$ is any fixed rearrangement invariant space from the class $\mathscr{L}$ (Def. 2.20). In the case when $X$ is an Orlicz space $L^{\phi}$, the symbol $L^{\Phi} \in \mathscr{L}$ means that $\Phi$ satisfies the condition $\Delta_{2}$ (see [6], p. 23). The necessary condition and the sufficient condition of this theorem are expressed as in the Shimogaki theorem, but by means of indices of Boyd and Zippin type (Th. 4.6).

In Section 3 of this paper there is described the $X$-Hardy property (Def. 3.1) which is a fundamental one for interpolation. There is also given a generalization of Hardy's inequality. Namely, if $\alpha(Y)<p_{0}(X)$ (where $p_{0}(X)$ denotes the lower Zippin index of $X$ ), then $\bar{Y}$ has $X$-Hardy property and

$$
\left\|\varphi_{X}(t)^{-1} \int_{0}^{t} f(s) d \varphi_{X}(s)\right\|_{Y} \leqslant \int_{0}^{1} h(s, Y) d M(s, X)\|f\|_{Y} .
$$

These considerations have been inspired by the paper by P. L. Butzer and F. Fehér [5] concerning, among other things, $L^{1 / 0}-\mathrm{H}$ ardy property $(0<\theta \leqslant 1)$ and Hardy's inequality; in this paper the following theorem is proved:

Theorem $\mathrm{B}$ (Boyd, see also Butzer-Fehér). A rearrangement invariant space $Y$ has the $L^{1 / \theta}-$ Hardy property if and only if $\alpha(Y)<\theta$. Moreover,

$$
\left\|t^{-\theta} \int_{0}^{t} s^{\theta-1} f(s) d s\right\|_{Y} \leqslant \int_{0}^{1} s^{\theta-1} h(s, Y) d s\|f\|_{Y} .
$$

Using Boyd's [2] considerations it is also proved that if $Y$ has the $X$-Hardy property, then $a(Y)<p_{\infty}(X)$. It can be scen that in case where $\varphi_{X}(t)=t^{\theta}$ then from the above facts we obtain Theorem $\mathrm{B}$ and inequality (1.2) as the particular case of (1.1).

In Section 5, a majorant for the class $C=\omega-\operatorname{Lip}\left(X ; K_{X}\right) \cap \operatorname{Lip}\left(L^{\infty} ; K_{\infty}\right)$ is found in the case where $\varphi_{X}(t)=t^{0}, 0<\theta \leqslant 1$. The general case remains open.

\$ 2. Rearrangement invariant spaces. Let $\mathscr{H}$ be the space of realvalued Lebesgue measurable functions on the interval $I=(0, l), 0<l$ $\leqslant \infty$. A Banach subspace $X$ of $\mathscr{M}$ satisfying ( $m$ denoting the Lebesgue measure)

(2.1) $|g| \leqslant|f|$ a.e., and $f \in X \quad$ implies $\quad g \in X$ and $\|g\|_{X} \leqslant\|f\|_{X}$;

(2.2) $\quad 0 \leqslant f_{n} \uparrow, \sup _{n \in N}\left\|f_{n}\right\|_{X} \leqslant \mathcal{I}$

$$
\text { implies } f=\sup _{n \in N} f_{n} \in X \text { and }\|f\|_{X}=\sup _{n \in N}\left\|f_{n}\right\|_{X}
$$

(2.3) $m A<\infty$ implies $\int_{A} f(s) d s \leqslant C_{A}\|f\|_{X}$,

with $C_{A}>0$ independent of $f \in X$;

(2.4) $m A<\infty$ implies $\left\|\chi_{d A}\right\|_{x}<\infty$, where $\chi_{A}$ is the characteristic function of tho set $A$

is usually referred to as a Banach function space (see [7], [9]). A Banach function space $X$ is called a rearrangement invariant (r.i.) space or symmetric in the terminology of Semenov if $d_{f}(y)=m\{s \in I:|f(s)|>y\}<\infty$ for each $f \in X, y>0$, and if

(2.5) for any $g \in \mathscr{M}$ equimeasurable to $f \in X$, i.e., $d_{g}=d_{f}$, one has $g \in X$ and $\|g\|_{X}=\|f\|_{X}$.

The nonincreasing rearrangement $f^{*}$ of a function $f \in X$ is an almost everywhere unique positive noninereasing function on $I$ which is equimeasurable to $|f|$. So, for an r.i. space we have

$$
\|f\|_{X}=\left\|f^{*}\right\|_{X}
$$

Examples of r.i. spaces include the Lebesgue $L^{p}$-spaces, the Orlicz $L^{\Phi}$ spaces and the Lorentz spaces $\Lambda, M$ and $L^{p q}$. Also if $X, Y$ are r.i. spaces, so is $X \cap Y, X+Y$, and $\bar{A}^{X}$ satisfy (2.1) and (2.5), where $A$ is symmetric linear subset of $X\left(A\right.$ is symmetric if $f \in A$ and $g^{*}(t) \leqslant f^{*}(t)$ imply $\left.g \in A\right)$.

We shall write $\langle f, g\rangle$ for the inner product $\int_{X} f(t) g(t) d t$.

The associated space $X^{\prime}$ of an r.i. space $X$ is defined by

$$
X^{\prime}=\left\{g \in \mathscr{M}:\|g\|_{X^{\prime}}=\sup _{\|f\|_{X} \leqslant 1}\langle|f|,|g|\rangle<\infty\right\} .
$$

It follows that $X^{\prime}$ is also an r.i. space under the norm $\|\cdot\|_{X^{\prime}}$. A result due to Lorentz and Luxemburg [9] is that $X$ is isometrically isomorphic to $X^{\prime \prime}$, and, in particular, the norm has the representation.

$$
\|f\|_{x}=\sup \left\{\left\langle f^{*}, g^{*}\right\rangle:\|g\|_{X^{\prime}} \leqslant 1\right\}
$$

from which the important Hölder inequality

follows.

$$
\left|\left\langle f, g_{1}\right\rangle\right| \leqslant\langle|f|,|g|\rangle \leqslant\left\langle f^{*}, g^{*}\right\rangle \leqslant\|f\|_{\boldsymbol{X}}\|g\|_{X^{\prime}}
$$


The smallest and the largest of the r.i. spaces are $L^{1} \cap L^{\infty}$ and $L^{1}+L^{\infty}$, respectively, in the sense that the continuous embeddings

$$
I^{1} \cap L^{\infty} \subset X \subset L^{1}+L^{\infty}
$$

hold for any r.i. space $X$.

The fundamental function $\varphi_{X}$ of an r.i. space $X$ is given by

$$
\varphi_{X}(t)=\left\|\chi_{(0, t)}\right\|_{X}, \quad t \in I .
$$

The fundamental function is a positive nondecreasing function on $I$ which is absolutely continuous on $[\varepsilon, l)$ for each $\varepsilon>0$ and satisfies

$$
\begin{aligned}
& \varphi_{X}(t) \varphi_{X^{\prime}}(t)=t \quad \text { for all } t \in I, \\
& \frac{d \varphi_{X}(t)}{d t} \leqslant \frac{\varphi_{X}(t)}{t} \quad \text { a.e. on } \mathrm{I} \text {. }
\end{aligned}
$$

A straightforward computation shows that (i) for the Orlicz space with Orliez norm $L^{\Phi}$ one has $\varphi_{L^{\Phi}}(t)=t \Psi^{-1}(1 / t)$, where $\Psi$ is complementary to the Orlicz function $\Phi$, (ii) for the Orlicz space with Luxemburg norm $L^{I \Phi}$ one has $\varphi_{I^{I \Phi}}(t)=1 / \Phi^{-1}(1 / t)$, (iii) $\varphi_{I^{\Phi}}$ is concave function, (iv) $\varphi_{I^{I q}}$ does not have to be concave function; it is sufficient to take $\Phi(u)=u^{2}$ for $0<u \leqslant 1$ and $u^{3}$ for $u>1$.

In [16] and [1.8] it was shown that $X$ can be equivalently renormed so that the resulting fundamental function is concave. Wo assume throughout this paper that $X$ is renormed in this manner, i.e., $\varphi_{X}$ is concave.

The rearrangement invariant Lorentz spaces associated with r.i. space $X$, defined by

$$
\Lambda(X)=\left\{f \in \mathscr{M}:\|f\|_{\Lambda(X)}=\int_{0}^{l} f^{*}(s) d \varphi_{X}(s)<\infty\right\},
$$

and

$$
M(X)=\left\{f \in \mathscr{M}:\|f\|_{M(X)}=\sup _{t \in I}\left(\frac{\varphi_{X}(t)}{t} \int_{0}^{t} f^{*}(s) d s\right)<\infty\right\},
$$

are extremal in the sense that

$$
\Lambda(X) \subset X \subset M(X)
$$

with normal continuous embeddings. We consider also a rearrangomont invariant quasi-Banach function space (Marcinkiewicz space)

$$
M^{*}(X)=\left\{f \in \mathscr{M}:\|f\|_{M^{*}(X)}=\sup _{t \in I}\left(\varphi_{X}(t) f^{*}(t)\right)<\infty\right\} .
$$

For example, if $X=L^{p q}$, then $\varphi_{X}(t)=t^{1 / p}$, so that $\Lambda(X)=L^{p 1}$ and $M^{*}(X)=L^{p \infty}$, the "wealk" $L^{p}$-spaces.
The fundamental Zippin indices of an r.i. space $X$ (see [18]) are given by

$$
\begin{aligned}
& p_{0}(X)=p_{0}(X, I)=\sup _{0<s<1} \theta(s)=\lim _{s \rightarrow 0^{+}} \theta(s), \\
& p_{\infty}(X)=p_{\infty}(X, I)=\inf _{s>1} \theta(s)=\lim _{s \rightarrow \infty} \theta(s),
\end{aligned}
$$

where $\theta(s)=\log M(s, X) / \log s$, and for $s>0$,

$$
M(s, X)=\sup _{t \in I, t s \in I}\left(\varphi_{X}(t s) / \varphi_{X}(t)\right) .
$$

If $m(s ; X)=\inf _{t \in I, t s \in I}\left(\varphi_{X}(t s) / \varphi_{X}(t)\right)$, then for each $\varepsilon>0$ there exists $\delta>0$ such that

$$
\begin{array}{ll}
s^{p_{0}(X)} \leqslant M(s, X) \leqslant s^{p_{0}(X)-s}, & 0<s<\delta, \\
s^{p_{\infty}(X)+s} \leqslant m(s, X) \leqslant s^{p_{\infty}(X)}, & 0<s<\delta .
\end{array}
$$

If $\tilde{X}$ is the space $X$ with an equivalent norm then $p_{0}(\tilde{X})=p_{0}(X)$ and $p_{\infty}(\tilde{X})=p_{\infty}(\tilde{X})$.

The upper and lower Boyd indices of an r.i. space $X$ (see [3]) are given by

$$
\begin{gathered}
\alpha(X)=\alpha(X, I)=\inf _{0<s<1} \theta_{1}(s)=\lim _{s \rightarrow 0^{+}} \theta_{1}(s), \\
\beta(X)=\beta(X, I)=\sup _{s>1} \theta_{1}(s)=\lim _{s \rightarrow \infty} \theta_{1}(s),
\end{gathered}
$$

where $\theta_{1}(s)=-\log h(s, X) / \log s$ and $h(s, X)$ is the operator norm on $X$ of the dilation operator

$$
\left(E_{s} f\right)(t)= \begin{cases}f(t s) & \text { if } \quad t s \in I \\ 0 & \text { elsewhere. }\end{cases}
$$

From the definition of $\alpha$ we have that for each $\varepsilon>0$ there is a $\delta>0$ such that

$$
s^{-\alpha(X)} \leqslant h(s, X) \leqslant s^{-\alpha(X)-\theta}, \quad 0<s<\delta .
$$

These indices are known to satisfy:

$$
\begin{gathered}
0 \leqslant \beta(X) \leqslant p_{0}(X) \leqslant p_{\infty}(X) \leqslant \alpha(X) \leqslant 1, \\
p_{0}\left(X^{\prime}\right)=1-p_{\infty}(X), \quad p_{\infty}\left(X^{\prime}\right)=1-p_{0}(X), \\
\beta\left(X^{\prime}\right)=1-\alpha(X), \quad \alpha\left(X^{\prime}\right)=1-\beta(X) .
\end{gathered}
$$

For familiar r.i. spaces such as the Lebesgue spaces $L^{p}, 1 \leqslant p \leqslant \infty$, the Orlicz space $L^{\Phi}$, the Lorentz spaces $\Lambda(\psi)$ and $M(\psi)$, both $p_{0}(\cdot)=\beta(\cdot)$ and $p_{\infty}(\cdot)=\alpha(\cdot)$ hold. T. Shimogaki [16] has given an example of r.i. spaces $X_{a}$ such that $p_{0}\left(X_{a}\right)=p_{\infty}\left(X_{a}\right)=a$ and $\beta\left(X_{a}\right)=0$ or $\alpha\left(X_{a}\right)=1$ for every $0<a<1$. 
The following class of spaces will be useful in this paper ([14]):

(2.20) $\quad X \in \mathscr{L}=\mathscr{L}(I)$, if for some $0<b<1$ there is a pair of positive constants $O$ and $\delta$ such that for $u, v \in I$

$$
\varphi_{X}(v) / \varphi_{\boldsymbol{X}}(u) \leqslant O(v / u)^{b} \quad \text { if } \quad v / u \leqslant \delta .
$$

Let us notice that $X \in \mathscr{L}$ if and only if $p_{0}(X)>0$; in the case of an Orlicz space, the condition $L^{\Phi} \in \mathscr{L}(I)$ is equivalent to the condition: $\Phi$ satisfies $\Delta_{2}(I)$ condition.

We shall use the following simple inequality repeatedly.

LEMnca (2.1). If $f_{1}(s), f_{2}(s)$ are nondecreasing absolutely continuous unctions such that $f_{1}(0)=f_{2}(0)=0$ and if $f_{1}(s) \leqslant f_{2}(s)$ for all $s \in I$, then for any nonnegative, nonincreasing function $g$

$$
\int_{I} g(s) d f_{1}(s) \leqslant \int_{I} g(s) d f_{2}(s) .
$$

8 3. $X$-Hardy property. Whereas in [3] and [5] the $L^{1 / \theta}$-Hardy property $(0<\theta \leqslant 1)$ is considered, we now want to replace $L^{1 / \theta}$ by any r.i. space $X$. The aim of this section is therefore to introduce the " $X$-Hardy property" and give necessary and sufficient conditions for this property to hold. For this purpose, let $X, Y$ denote any r.i. spaces on $I$ with $X \in \mathscr{L}$, and $[Y]$ the space of bounded linear operators from $Y$ into itself. by

We define an operator $P_{X}$ associated with an r.i. space $X$ for $f \in \mathscr{M}$

$$
\left(P_{X} f\right)(t):=\varphi_{X}(t)^{-1} \int_{0}^{t} f(s) d \varphi_{X}(s), \quad t \in I
$$

whenever the integral exists a.e.

Definimion (3.1). We shall say that $Y$ has the $X$-Hardy property (we write $Y \in \mathrm{HP}_{X}$ ), if the operator $P_{X} \in[Y]$.

If $P_{X} \in[Y]$ and $|\lambda|<r\left(P_{X}\right)^{-1}$, where $r\left(P_{X}\right)$ denotes the spectral radius of the operator $P_{X}$, and $I$ is the identity operator, then $\left(I-\lambda P_{X}\right)^{-1}$ exists and $\left(I-\lambda P_{X}\right)^{-1} \in[Y]$, and

$$
\left(I-\lambda P_{X}\right)^{-1}=\sum_{n=0}^{\infty} \lambda^{n} P_{X}^{n}
$$

where the series converges in operator norm, and $P_{X}^{n}$ denotes the $n$th iterate of $P_{X}$. Since

$$
\left(P_{X}^{n} f\right)(t)=\varphi_{X}(t)^{-1} \int_{0}^{1} \frac{1}{(n-1) !}\left(\ln \frac{\varphi_{X}(t)}{\varphi_{X}(t s)}\right)^{n-1} f(s t) d \varphi_{X}(t s)
$$

one has

$$
\begin{aligned}
& \left(P_{X(\lambda)} f\right)(t):=P_{X}\left(I-\lambda P_{X}\right)^{-1} f(t) \\
& =\varphi_{X}(t)^{-1} \int_{0}^{1} f(s t)\left(\frac{\varphi_{X}(t)}{\varphi_{X}(t s)}\right)^{\lambda} d \varphi_{X}(t s)
\end{aligned}
$$

LEIDMA (3.2). If $P_{X(\lambda)} \in[Y]$, and $\lambda>0$, then for every $\varepsilon>0$ there exists $\delta>0$ such that for every $u \in(0, \delta)$

$$
h(u, Y) \int_{0}^{u} M \Gamma(s, X)^{-\lambda} d s^{p_{\infty}(X)+-s} \leqslant\left\|P_{X(\lambda)}\right\|_{[X]} .
$$

Proof (see [1]). Since $P_{X(\lambda)} \in[Y]$ and $s^{p_{\infty}(X)+s} \leqslant \varphi_{X}(t s) / \varphi_{X}(t)$ for $0<s<\delta$, we have for every nonnegative and nonincreasing $f, g$

$$
\begin{array}{rl}
\left\langle E_{u} f, g\right\rangle \int_{0}^{u} M & M(s, X)^{-\lambda} d s^{p_{\infty}(X)+e} \leqslant \int_{0}^{u} M(s, X)^{-\lambda}\left\langle E_{s} f, g\right\rangle d s^{p_{\infty}(X)+s} \\
& \leqslant \int_{0}^{u}\left(\varphi_{X}(t s) / \varphi_{X}(t)\right)^{-\lambda}\left\langle E_{s} f, g\right\rangle d s^{p_{\infty}(X)+s} \\
& =\left\langle\int_{0}^{u}\left(\varphi_{X}(\cdot s) \mid \varphi_{X}(\cdot)\right)^{-\lambda} f(\cdot s) \chi_{I}(\cdot s) d s^{p_{\infty}(X)+s}, g\right\rangle \\
& \leqslant\left\langle\int_{0}^{u}\left(\varphi_{X}(\cdot s) \mid \varphi_{X}(\cdot)\right)^{-\lambda} f(\cdot s) \chi_{I}(\cdot s) d\left(\varphi_{X}(\cdot s) / \varphi_{X}(\cdot)\right), g\right\rangle \\
& \leqslant\left\langle P_{X(\lambda)} f, g\right\rangle \leqslant\left\|P_{X(\lambda)} f\right\|_{Y}\|g\|_{X^{\prime}} \leqslant\left\|P_{X(\lambda)}\right\|_{[Y]}\|f\|_{Y}\|g\|_{X^{\prime}} \cdot
\end{array}
$$

Taking the supremum over all $g \in \dot{X}^{\prime},\|g\|_{Y^{\prime}} \leqslant 1$, and then over all $f \in Y$, $\|f\|_{X} \leqslant 1$, we obtain (3.5).

Lamra (3.3). Let $p_{0}(X)>0$. The following assertions are equivalent:

$$
\text { (3.6) } \quad \alpha(X)<p_{0}(X) \text {; }
$$

(3.7) There exist positive numbers $K$ and $\gamma(0 \leqslant \gamma<1)$ such that $h(s, Y)$ $\leqslant K s^{1-p_{0}(X)-\gamma}, 0<s \leqslant 1$

(3.8) $\quad s^{p_{0}(X)} h(s, Y) \rightarrow 0$ as $s \rightarrow 0^{+}$;

(3.9) $\quad A_{p_{0}(X)}:=\int_{0}^{1} s^{p_{0}(X)-1} h(s, X) d s<\infty$;

(3.10) $\quad \sigma_{0}(X, Y):=\int_{0}^{1} h(s, Y) d M(s, X)<\infty$.

Proof. For $(3.6) \Leftrightarrow(3.7) \Leftrightarrow(3.8) \Leftrightarrow(3.9)$ see [1] and [5]. 
$(3.6) \Rightarrow(3.10)$ : Let us take $\varepsilon>0$ such that $a(Y)+2 \varepsilon<p_{0}(X)$, then by Lemma (2.1)

$$
\begin{aligned}
C_{0}(X, Y) & \leqslant \int_{0}^{\delta} s^{-a(X)-\varepsilon} d M(s, X)+\int_{\delta}^{1}(1 / s) d M(s, X) \\
& \leqslant \int_{0}^{\delta} s^{-a(Y)-8} d s^{p_{0}(X)-s}+\text { const }<\infty .
\end{aligned}
$$

$(3.10) \Rightarrow(3.6)$ : Evidently since by Lemma (2.1)

$$
0<\int_{0}^{\delta} s^{-\alpha(Y)} d s^{p_{0}(X)} \leqslant \int_{0}^{\delta} s^{-\alpha(X)} d M(s, X) \leqslant O_{0}(X, Y) .
$$

THEOREM (3.4). (a) If $C_{0}(X, Y)<\infty$, then $P_{X} \in[Y]$ and $\left\|P_{X}\right\|_{[Y]}$ $\leqslant C_{0}(X, Y)$

(b) If $P_{X} \in[Y]$, then $A_{p_{\infty}(X)}:=\int_{0}^{1} s^{p_{\infty}(X)-1} h(s, Y) d s<\infty$.

Proof. (a): Using the Fubini theorem, Lemma (2.1) and the Földer inequality, we have

$$
\begin{aligned}
\left\langle\left(P_{X} f\right)^{*}, g^{*}\right\rangle \leqslant\left\langle P_{X} f^{*}, g^{*}\right\rangle=\int_{0}^{l}\left(\varphi_{X}(t)^{-1} \int_{0}^{1} f^{*}(t s) d \varphi_{X}(t s)\right) g^{*}(t) d t \\
\quad \leqslant \int_{0}^{1}\left(\int_{0}^{l} E_{s} f^{*}(t) g^{*}(t) d t\right) d M(s, X) \\
\quad \leqslant \int_{0}^{1}\left\|E_{s} f^{*}\right\|_{Y}\left\|g^{*}\right\|_{Y^{\prime}} d M(s, X) \leqslant \int_{0}^{1} h(s, Y) d M(s, X)\left\|f^{*}\right\|_{Y}\left\|g^{*}\right\|_{X^{\prime}}
\end{aligned}
$$

Taking the supremum over all $g \in Y^{\prime},\|g\|_{Y^{\prime}} \leqslant 1$, we obtain by $(2.6)$ the generalized inequality of the Hardy type:

$$
\left\|\varphi_{X}(t)^{-1} \int_{0}^{t} f(s) d \varphi_{X}(s)\right\|_{X} \leqslant \int_{0}^{1} h(s, X) d M(s, X)\|f\|_{Y} .
$$

(b): Let $0<\lambda<1$ and $\lambda<r\left(P_{\tilde{X}}\right)^{-1}$. Then $P_{X(\lambda)} \in[Y]$ and, : by Lemma (3.2),

i.e.

$$
h(u, Y) \int_{0}^{u} M(s, X)^{-\lambda} d s^{p_{\infty}(X)+e} \leqslant\left\|P_{X(\lambda)}\right\|_{[X]},
$$

$$
h(u, Y) \leqslant \text { const } u^{-\left(p_{\infty}(X)+\varepsilon-\lambda p_{0}(X)+\varepsilon \lambda\right)} \text { for } u \in(0, \delta) .
$$

Taking $\varepsilon<\lambda p_{0}(X) /(\mathbf{1}+\lambda)$, we obtain

$$
\begin{aligned}
A_{p_{\infty}(X)} \leqslant \text { const } \int_{0}^{s} s^{p_{\infty}(X)-1} s^{-\left(p_{\infty}(X)-\lambda p_{0}(X)+s+e \lambda\right)} d s+ \\
+\operatorname{const} \int_{d}^{1} s^{p_{\infty}(X)-2} d s<\infty .
\end{aligned}
$$

According to Tomma (3.3), Theorem (3.4), and fact that $A_{p_{\infty}(X)}<\infty$ implies $\alpha(X)<p_{\infty}(X)$, wo obtain

CononianX (3.5). If $a(X)<p_{0}(X)$, then $X \in \mathrm{Tr}_{X} ;$ if $Y \in \mathrm{HP}_{X}$, then $\alpha(X)<p_{\infty}(X)$.

Irence the following follows directly:

Coroxtadi (3.6). Let $p_{0}(X)=p_{\infty}(X)>0 . Y \in \mathrm{HT}_{X}$ if and only if $\alpha(X)<p_{0}(X)$.

In particular, if $\varphi_{X}(t)=t^{\theta}, 0<\theta \leqslant 1$, then $Y \in \operatorname{IrP}_{X}$ if and only if $\alpha(X)<\theta$ (Theorem $\mathbf{B})$.

Exancote (3.7). We consider Orlicz space $L \log ^{+} L$ on $(0,1)$ generated by the Orlicz function $\Phi(u)=u \log ^{+*} u$ with Orlicz norm. Then

$$
\begin{aligned}
& \varphi_{L \log +L}(t)=t(1-\log t), \quad p_{0}\left(L \log ^{+} L\right)=1, \\
& \left(P_{x \log +x} f\right)(t)=[t(1-\log t)]^{-1} \int_{0}^{t} f(s) \log 1 / s d s,
\end{aligned}
$$

and

$$
C_{0}\left(L \log ^{+} I, Y\right)=\int_{0}^{1} h(s, Y) \log 1 / s d s .
$$

By Oorollary (3.6), $X \in \mathbb{E P}_{L \log +\infty}$ if and only if $\alpha(Y)<1$.

OONJEOCURE (3.8). There exist r.i. spaces $X_{i}, Y_{i}$ such that $0<p_{0}\left(X_{i}\right) \leqslant \alpha\left(Y_{i}\right)<p_{\infty}\left(X_{i}\right)(i=1,2)$, and $Y_{1} \notin \mathrm{HP}_{X_{1}}$, and $Y_{2} \in \mathrm{HT}_{\Lambda_{2}}$.

\$ 4 . Interpolation of Lipschitz operators of weak type $(X, X)$ and type $\left(L^{\infty}, L^{\infty}\right)$. Let $\left(X_{1}, X_{2}\right)$ be a quasi-Banach couple, i.e., there is a Hausdorff topologioal rector space $\mathfrak{X}$ and continuous embeddings $X_{1} \subset \mathfrak{X}, \mathrm{X}_{2} \subset \mathfrak{X}$.

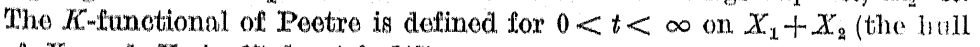
of $X_{1}$ and $X_{2}$ in $x^{*}$ ) by (of. [4])

(4.1) $\quad K(t, f)=K\left(t, f ; X_{1}, X_{2}\right)$

$$
=\inf \left\{\left\|f_{1}\right\|_{x_{1}}+\|\|_{f_{2}} \|_{x_{2}} ; f_{1} \in X_{1}, f_{2} \in X_{2}, f=f_{1}+f_{2}\right\} \text {. }
$$

Wo sissume throughout this section as well as the rest of the paper that $X \in \mathscr{L}$ has a striotly increasing concave fundamental function $\varphi_{X}$; this is no restriction if $l=\infty$. 
Since $X \in \mathscr{L}$, we have $\lim _{t \rightarrow 0^{+}} \varphi_{\mathbf{X}}(t)=0$, and therefore

(4.2) $\quad K\left(t, f ; \Lambda(X), L^{\infty}\right)=\int_{0}^{\min \left(\varphi_{X}^{-1}(t), l\right)} f^{*}(s) d \varphi_{X^{\prime}}(s) \quad$ (see [14]).

For $a>0, f^{(a)}$ will denote the a-truncation of $f$, that is,

$$
f^{(\mathfrak{a})}(x)=\min (|f(x)|, \mathfrak{a}) \operatorname{sgn} f(x) .
$$

Learaca (4.1). We have

$$
\frac{1}{2} \sup _{s \in S_{t}} \varphi_{X}(s) f^{*}(s) \leqslant K\left(t, f ; M^{*}(X), L^{\infty}\right) \leqslant 2 \sup _{s \in S_{t}} \varphi_{X}(s) f^{*}(s),
$$

where

$$
S_{t}=\left\{s \in I: s \leqslant \min \left(\varphi_{X}^{-1}(t), l\right)\right\} .
$$

Proof. If $l<\infty$, then $\|f\|_{M^{*}(X)} \leqslant \varphi_{X}(l)\|f\|_{L^{\infty}}$ for $f \in L^{\infty}$, so $\frac{1}{2}\|f\|_{M^{*}(X)}$ $\leqslant K(t, f) \leqslant\|f\|_{M^{*}(X)}$ for $t \geqslant \varphi_{X}(l)$. Let $\varphi_{X}^{-1}(t)<l$ and $\mathfrak{a}=f^{*}\left(\varphi_{X}^{-1}(t)\right)$ and let $f_{1}=f-f^{(a)}, f_{2}=f^{(a)}$. Since $\left(f-f^{(a)}\right)^{*}=f^{*}-\left(f^{*}\right)^{(a)}$, we have

$$
\begin{aligned}
K(t, f) & \leqslant\left\|f_{1}\right\|_{M^{*}(X)}+t\left\|f_{2}\right\|_{L^{\infty}} \\
& \leqslant \sup _{s \in I} \varphi_{X}(s)\left[f^{*}(s)-\left(f^{*}\right)^{(\mathfrak{a})}(s)\right]+t f^{*}\left(\varphi_{X}^{-1}(t)\right) \\
& =\sup _{0<s \leqslant q_{X}^{-1}(t)} \varphi_{X}(s)\left[f^{*}(s)-f^{*}\left(\varphi_{X}^{-1}(t)\right)\right]+t f^{*}\left(\varphi_{X}^{-1}(t)\right) \\
& \leqslant 2 \sup _{0<s \leqslant \varphi^{-1}(t)} \varphi_{X}(s) f^{*}(s) .
\end{aligned}
$$

On the other hand, let $\varepsilon \in(0,1)$ be an arbitrary fixed number, and let $f=f_{1}+f_{2}$ be an arbitrary decomposition of $f \in M^{*}(X)+L^{\infty}$. Then

$$
\begin{aligned}
\left\|f_{1}\right\|_{M^{*}(X)}+t\left\|f_{2}\right\|_{L^{\infty}} & \geqslant \sup _{0<s \leqslant(1-\varepsilon) \varphi_{X}^{-1}(t)} \varphi_{X}(s) f_{1}^{*}(s)+t \sup _{0<s \leqslant s \varphi_{X}^{-1}(t)} f_{2}^{*}(s) \\
& =\sup _{0<v \leqslant \varphi_{X}^{-1}(t)} \varphi_{X}((1-\varepsilon) v) f_{1}^{*}((1-\varepsilon) v)+\sup _{0<v \leqslant \varphi_{X}^{-1}(t)} \varphi_{X}(v) f_{2}^{*}(\varepsilon v) \\
& \geqslant(1-\varepsilon) \sup _{0<v \leqslant \varphi_{X}^{-1}(t)} \varphi_{X}(v) f_{1}^{*}((1-\varepsilon) v) \\
& +\sup _{0<v \leqslant \varphi_{X}^{-1}(t)} \varphi_{X}(v) f_{2}^{*}(\varepsilon v) \geqslant(1-\varepsilon) \sup _{0<v \leqslant \varphi_{X}^{-1}(t)} \varphi_{X}(v) f^{*}(v) .
\end{aligned}
$$

Since the decomposition and $\varepsilon>0$ are arbitrary, we obtain the left-hand inequality.

LeMMA (4.2). If $p_{\infty}(Y)<p_{0}(X)$, then $Y$ is continuously embedded in $\Lambda(X)+L^{\infty}$.
Proof. Since

$$
f^{*}(s) \leqslant s^{-1} \int_{0}^{\infty} f^{*}(t) d t \leqslant \varphi_{Y}(s)^{-1}\|f\|_{M(X)} \quad \text { for all } s \in I,
$$

we have

$$
\begin{aligned}
\|f\|_{\Lambda(X)+L^{\infty}} & =K\left(1, f ; \Lambda(X), L^{\infty}\right)=\int_{0}^{\min \left(p_{X}^{-1}(1), l\right)} f^{*}(s) d p_{X}(s) \\
& \leqslant \int_{0}^{\min \left(\varphi_{X}^{-1}(1), l\right)} \varphi_{Y}(s)^{-1} d \varphi_{X}(s)\|f\|_{M(X)} .
\end{aligned}
$$

By relations (2.11), (2.13), and (2.14) we obtain

$$
\begin{aligned}
& \int_{0}^{\min \left(\varphi_{X}^{-1}(1), l\right)} \varphi_{X}(s)^{-1} d \varphi_{X}(s) \leqslant \int_{0}^{\min \left(\varphi_{X}^{-1}(1), l\right)} \varphi_{Y}(s)^{-1} \varphi_{X}(s) d s / s \\
& \leqslant \text { const }\left(\int_{0}^{s} s^{p_{0}(X)-\varepsilon-\left(p_{\infty}(X)+\varepsilon\right)} d s / s+\int_{0}^{\min \left(\varphi_{X}^{-1}(1), l\right)} \varphi_{Y}(s)^{-1} \varphi_{X}(s) d s / s\right)<\infty .
\end{aligned}
$$

By (2.12), we have $X \subset \Lambda(X)+L^{\infty}$.

We denote $K_{0}\left(t, f ; M^{*}(X), L^{\infty}\right)=\sup _{s \in S_{t}} \varphi_{X}(s) f^{*}(s)$.

A rearrangement invariant space $Y, X \subset \Lambda(X)+L^{\infty}$, is called $K_{0^{-}}$ monotone with regard to couples $\left(\Lambda(X), L^{\infty}\right),\left(M^{*}(X), L^{\infty}\right)$, if $f \in \bar{Y}$, $g \in M^{*}(X)+L^{\infty}, K_{0}\left(t, g ; M^{*}(X), L^{\infty}\right) \leqslant K\left(t, f ; A(X), L^{\infty}\right)$ for $t>0$, imply $g \in Y$ and $\|g\|_{Y} \leqslant O\|f\|_{Y}$ with some $O=C(Y)>0$.

LEvara (4.3). $Y$ is $K_{0}$-monotone with regard to couples $\left(\Lambda(X), L^{\infty}\right)$, $\left(M^{*}(X), L^{\infty}\right)$ if and only if $Y \in \mathrm{HP}_{X}$.

Proof. The necessity follows from the fact that

$$
\begin{aligned}
& K_{0}\left(t, P_{X} f ; M^{\star}(X), L^{\infty}\right) \leqslant \sup _{s \in S_{t}} \varphi_{X}(s)\left(P_{X} f^{\star}\right)(s) \\
& { }_{\operatorname{s\in S} S_{t}}^{\min \left(\varphi_{X}^{-1}(l), r\right)} \\
& =\int_{0}^{(x)} f^{*}(s) d \varphi_{X}(s)=K\left(t, f ; A(X), x^{\infty}\right) .
\end{aligned}
$$

Concerning the sufficiency, for $t>0$ such that $\varphi_{\mathbf{X}}^{-1}(t)<l$ we have

$$
\begin{aligned}
t^{*}\left(\varphi_{X}^{-1}(t)\right) & \leqslant \sup _{0<<<<\varphi_{X}^{-1}(t)} \varphi_{X}(s) g^{*}(s) \\
& =K_{0}\left(t, g ; M^{*}(X), L^{\infty}\right) \leqslant K\left(t, f ; \Lambda(X), L^{\infty}\right) .
\end{aligned}
$$

Fence

$$
g^{*}(u) \leqslant \varphi_{X}(u)^{-1} \int_{0}^{u} f^{*}(s) d \varphi_{X}(s)=\left(P_{X} f^{*}\right)(u)
$$


LEMMa (4.4). If $T \in \omega-\operatorname{Lip}(X ; 1) \cap \operatorname{Lip}\left(L^{\infty} ; 1\right)$, then $K_{0}\left(t, T f ; M^{*}(X)\right.$, $\left.L^{\infty}\right) \leqslant K\left(t, f ; \Lambda(X), L^{\infty}\right)$ for $t>0$ and $f \in \Lambda(\ddot{X})$.

Proof. For each $t>0$ we put $\mathfrak{a}=f^{*}\left(\min \left(\varphi_{X}^{-1}(t), l\right)\right)$. We have

$$
K_{0}\left(t, T f ; M^{*}(X), L^{\infty}\right)
$$

$$
\begin{aligned}
& \leqslant \sup _{s \in S_{t}} \varphi_{X}(s)\left\{(T f)^{*}(s)-\left[(T f)^{*}\right]^{(\mathfrak{a})}(s)\right\}+\sup _{s \in S_{t}} p_{X}(s)\left[(T f)^{*}\right]^{(\mathfrak{a})}(s) \\
& \leqslant\left\|T f-(T f)^{(\mathfrak{a})}\right\|_{M^{*}(X)}+t^{\prime} \mathfrak{a}, \quad \text { where } \quad t^{\prime}=p_{X}\left(\min \left(p_{X}^{-1}(t), l\right)\right) .
\end{aligned}
$$

Since $T \in \operatorname{Lip}\left(L^{\infty} ; 1\right)$, we get $\left\|T\left(f^{(\mathfrak{a})}\right)\right\|_{L^{\infty}} \leqslant\left\|f^{(\mathfrak{a})}\right\|_{L^{\infty}} \leqslant \mathfrak{a}$, which implies

(4.4.)

$$
\left|T f-(T f)^{(a)}\right| \leqslant\left|T f-T\left(f^{(\mathrm{a})}\right)\right| .
$$

Therefore we obtain

$$
\begin{aligned}
K_{0}\left(t, T f ; M^{*}(X), L^{\infty}\right) \leqslant\left\|T f-T\left(f^{(\mathfrak{a})}\right)\right\|_{M^{*}(X)}+t^{\prime} \mathfrak{a} \\
\leqslant\left\|f-f^{(\mathfrak{a})}\right\|_{A(X)}+t^{\prime} \mathfrak{a}=\int_{0}^{\min \left(\omega_{X}^{-1}(t), l\right)}\left(f^{*}(s)-\mathfrak{a}\right) d \varphi_{X}(s)+t^{\prime} \mathfrak{a} \\
=\int_{0}^{\min \left(\varphi_{X}^{-1}(t), l\right)} f^{*}(s) d \varphi_{X}(s)=K\left(t, f_{\circ} ; \Lambda(X), L^{\infty}\right) .
\end{aligned}
$$

This lemma can be proved also as in [15] by means of the fact that

$$
\|f\|_{M^{*}(X)}=\sup _{y>0} y \varphi_{X}^{-1}\left(d_{f}(y)\right) .
$$

In $[17]$, p. 215 , one can find the proof of $(4.5)$ in the case $X=L^{p}$; the proof for any $X \in \mathscr{L}$ is similar.

THEOREM (4.5). Let $l<\infty$. $Y$ has the interpolation property for the class $\omega$-Lip $\left(X ; K_{X}\right) \cap \operatorname{Lip}\left(L^{\infty} ; K_{\infty}\right)$ if and only if $Y \in \mathrm{HP}_{X}$.

Proof. Sufficiency. Suppose that $Y \in \mathrm{HP}_{X}$ and let $T \in \omega$ - $\operatorname{Lip}\left(X ; K_{X}\right)$ $\cap \operatorname{Lip}\left(L^{\infty} ; K_{\infty}\right)$. We fix $h \in \Lambda(X) \cap L^{\infty}$, and define the operator $S$ by

$$
S f=\frac{T(f+h)-T h}{\max \left(K_{X}, K_{\infty}\right)}, \quad f \in \Lambda(X) \cup L^{\infty}
$$

Then $S \in \omega-\operatorname{Lip}(X ; 1) \cap \operatorname{Lip}\left(L^{\infty} ; 1\right)$. Hence, by Lemma $(4: 4), K_{0}(t, S f)$; $\left.M^{*}(X), L^{\infty}\right) \leqslant K\left(t, f ; \Lambda(X), L^{\infty}\right)$, and by Lomma (4.3), $\|S f\|_{Y} \leqslant\left\|P_{X}\right\|_{[Y]}$ $\|f\|_{Y}$. This means that

$$
\|T f-T h\|_{Y}=\max \left(K_{X}, K_{\infty}\right)\|S(f-h)\|_{Y} \leqslant \max \left(K_{X}, K_{\infty}\right)\left\|P_{X}\right\|_{[Y]}\|f-h\|_{1^{*}}
$$

for $f \in \Lambda(X) \cup L^{\infty}, h \in \Lambda(X) \cap L^{\infty}$. For arbitrary $f, g \in Y$ wo consider the truncations $f^{(n)}, g^{(n)}$. Then $T\left(f^{(n)}\right)$ and $T\left(g^{(n)}\right)$ converge in the $M^{*}(X)$ quasi-norm to $T f$ and $T g$, respectively, since $T \in \omega$-Lip $\left(X ; K_{X}\right)$ and $Y \subset \Lambda(X)$. Therefore, for a properly chosen sequence $\left(n_{i}\right), T\left(f^{\left(n_{i}\right)}\right)$ and
$T\left(g^{\left(n_{i}\right)}\right)$, converge almost everywhere to $T f$ and $T g$. Since $f^{(n)} \in L^{\infty}$ and $\left|f^{(n)}-g^{(n)}\right| \leqslant|f-g|$, we obtain

$$
\left\|T\left(f^{\left(n_{i}\right)}\right)-T\left(g^{\left(n_{i}\right)}\right)\right\|_{Y} \leqslant \max \left(K_{X}, K_{\infty}\right)\left\|P_{X}\right\|_{[Y]}\|f-g\|_{Y} .
$$

Hence, by virtue of property (2.2),

$$
\|T f-T g\|_{Y} \leqslant \max \left(K_{X}, K_{\infty}\right)\left\|P_{X}\right\|_{[Y]}\|f-g\|_{Y}, \quad f, g \in X .
$$

Neoessity. This follows from the fact that

$$
\text { (4.7) " } \quad P_{X} \in \omega-\operatorname{Lip}(X ; 1) \cap \operatorname{Lip}\left(L^{\infty} ; 1\right) .
$$

Remark. Since (4.7) is also true for $l=\infty$, the necessary condition of the Theorem 4.5 includes the case $l=\infty$

Theorem (4.5) and Corollary (3.5) imply

THEorem (4.6). (a) Let $l<\infty$. If $\alpha(Y)<p_{0}(X)$, then $Y$ has the interpolation property for the class $\omega$-Lip $\left(X ; K_{X}\right) \cap \operatorname{Lip}\left(L^{\infty} ; K_{\infty}\right)$ and

$$
\|T\|_{\left[Y_{]}\right]} \leqslant \max \left(K_{X}, K_{\infty}\right)\left\|P_{X}\right\|_{[Y]} .
$$

(b) If $Y$ has the interpolation property for the class $\omega$-Lip $\left(X ; K_{X}\right) \cap$ $\cap \operatorname{Lip}\left(L^{\infty} ; K_{\infty}\right)$, then $\alpha(Y)<p_{\infty}(X)$.

Goroliani (4.7). Let $l<\infty$ and let $p_{\infty}(X)=p_{0}(X)>0 . Y$ has the interpolation property for the class $\omega-\operatorname{Lip}\left(X ; K_{X}\right) \cap \operatorname{Lip}\left(L^{\infty} ; K_{\infty}\right)$ if and only if $\alpha(Y)<p_{0}(X)$.

If the norm of $Y$ is continuous, that is $0 \leqslant f_{n} \downarrow 0$ implies $\left\|f_{n}\right\| \downarrow 0$, we can extend Theorems (4.5) and (4.6) to spaces on $(0, \infty)$. In fact, we have

Truendin (4.8). Let $l=\infty$ and let the norm on $Y$ be continuous. Then the statements of sufficiency of Theorems (4.5) and (4.6) remain valid if $T$ is replaced by the unique extension of $T$ onto $Y$.

The proof is derived from the facts that $T$ is also a Lipschitz operator from $Z=X \cap \Lambda(X) \cap L^{\infty}$ (with the norm of $Y$ ) into $Y$, and $Z$ is dense in $Y$.

In particular, in case $X=L^{1}$ by Corollary (4.7) and Theorem (4.8) wo have Theorem $A$.

\$5. Majorant for interpolation theorem. By Theorem (4.6) wo seo that each operator $T$ of the class

$$
C=\omega-\operatorname{Lip}\left(X ; K_{X}\right) \cap \operatorname{Lip}\left(L^{\infty} ; K_{\infty}\right)
$$

is a lipschitz operator on $Y$ with the bound not exceeding $\max \left(\boldsymbol{K}_{X}\right.$, $\left.K_{\infty}\right)\left\|P_{X}\right\|_{[X]}$ if $\alpha(X)<p_{0}(X)$. In [15] it is shown that if $T \in \omega-\operatorname{Lip}\left(L^{1} ; K_{1}\right) \cap$ $\cap \operatorname{Lip}\left(L^{\infty} ; K_{\infty}\right)$ and $\alpha(Y)<1$, then $\|T\|_{\text {Lip }[Y]} \leqslant K_{\infty} h(a, Y)\left\|P_{L^{1}}\right\|_{[Y]}$, where $a=K_{\infty} K_{1}^{-1}$. For the class $\omega-\operatorname{Lip}\left(X ; K_{X}\right) \cap \operatorname{Lip}\left(L^{\infty} ; K_{\infty}\right)$ we have

6 - Studia Mathematica 71 
THEOREMr. (5.1). (a) If $\alpha(Y)<p_{0}(X)$, then

(5.1) $\quad\|T\|_{\text {Lip }[Y]} \leqslant K_{\infty} h(a, Y)\left\|P_{X}\right\|_{[Y]}$, where $a=n\left(K_{\infty} K_{X}^{-1}, X\right)$

and

$$
n(s, X)=\inf _{t \in I_{\varphi}, t s \in I_{\varphi}} \frac{\varphi_{\bar{X}}^{-1}(t s)}{\varphi_{\bar{X}}^{-1}(t)}, \quad I_{\varphi}=(0, \varphi(l)) .
$$

Proof. Since $\varphi_{X}(n(s, X) u) \leqslant s \varphi_{X}(u)$, for any $T \in O$ the operator $T^{\prime}=K_{\infty}^{-1} E_{\alpha^{-1}} T$ belongs to $\omega-\operatorname{Lip}(X ; 1) \cap \operatorname{Lip}\left(L^{\infty} ; 1\right)$ if $0<a<1$, and the operator $T^{\prime \prime}=K_{\infty}^{-1} T E_{a^{-1}}$ belongs to $\omega-\operatorname{Lip}(X ; 1) \cap \operatorname{Lip}\left(L^{\infty} ; 1\right)$ if $a \geqslant 1$. Now, 5.1 is proved in the same manner as in [8].

For example, if $\varphi_{X}(t)=t^{0}, 0<\theta \leqslant 1$ and $\alpha(Y)<\theta$, then

(5.2) $\|T\|_{\text {Lip }[Y]} \leqslant K_{\infty} h(a, Y)\left\|P_{X}\right\|_{[Y]}$, where $\quad a=\left(K_{\infty} \cdot K_{X}^{-1}\right)^{1 / \theta}$

and

$$
\sup \left\{\|T\|_{L i p[Y]}: T \in C\right\}=K_{\infty} h(a, Y)\left\|P_{X}\right\|_{[X]} .
$$

Acknowledgements. The author wishes to thank Professor W. Orlicz for his valuable suggestions and very. helpful comments that made possible the preparation of this paper. The author also expresses his sincere gratitude to Professor P. L. Butzer and Doctor F. Feher who read the manuscript and gave many important comments.

Added in proof. After this paper was submitted for publication, the author answered Conjecture (3.8) in the affirmative, see Indices and interpolation, preprint.

\section{References}

[1] D. W. B oyd, The Hilbert transform on rearrangement-invariant spaces, Canad. J. Math. 19 (1967), 599-616.

[2] - The spectral radius of averaging operators, Pacific J. Math. 24 (1968), 19-28.

[3] - Indices of function spaces and their relationship to interpolation, Canad. J. Math. 21 (1969), 1245-1254.

[4] P. L. Butzer and H. Berens, Semi-groups of operators and approximation, Grundlehren Math. Wiss. 145, Berlin 1967.

[5] P. L. Butzer and F. Fehér, Generalized Hardy and Hardy-Littlewood inequalities in rearrangement invariant spaces, Comment. Math. Prace Mat. Tomus Specialis in Honorem Ladislai Orlicz I (1978), 41-64.

[6] M. A. Krasnosel'skii and Ya. B. Rutickii, Oonvex funotions and Orlice spaces, Noordhoff, Groningen 1961.

[7] G. G. Lorentz, Bernstein polynomials, University of Toronto Press, Toronto 1953.

[8] G. G. Lorentz and T. Shimogaki, Majorants for interpolation theorems, Publ. Ramanujan Inst. 1 (1969), 115-122.

[9] W. A. J. Luxemburg, Banach function spaces, Thesis, Van Goroum, Assen

1955.
[10] L. Maligranda, Interpolation of Lipsehitz operators for the pairs of spaces $\left(L^{1}, L^{\infty}\right)$ and $\left(l^{1}, c_{0}\right)$, Comment. Math. Prace Mat. 21 (1979), 327-339.

[11] - Interpolation of Lipsehitz operators for the pairs of spaces $\left(L^{p}, L^{\infty}\right)$ and $\left(l^{p}, c_{0}\right)$, $0<p<\infty$, Funct. Approximatio 9 (1980), 107-115.

[12] W. Matuszewska and W. Orlicz, On certain properties of p-functions, Bull. Acad. Polon. Sci. Sér. Sci. Math. Astronom. Phys. 8 (1960), 439-443.

[13] W. Orlicz, On a class of operations over the space of integrable functions, Studia Math. 14 (1954), 302-309.

[14] R. C. Sharpley, Spaces $A_{a}(X)$ and interpolation, J. Functional Analysis 11 (1972), 479-513.

[15] T. Shimogaki, An interpolation theorem on Banach funotion spaces, Studia Math. 31 (1968), 233-240.

[16] - Indices of function spaces, preprint.

[17] E. Stein and G. Weiss, Introduotion to Fourier analysis on Evelidean spaces (Russian), Mir, Moseow 1974.

[18] M. Zippin, Interpolation of operators of weak type between rearrangement ittvariant function spaces, J. Functional Analysis 7 (1971), 267-284.

INSTITUT OF MATHEMATICS

POLISH ACADEMY OF SOIENCES, BRANCH IN POZNAN

INSTYTUT MATEMATYOZNY PAN, ODDZTAE W POZNANIU

Reoeived Maroh 5, 1979 